\title{
Systematically Bridging the Gap between Novæ and Supernovæ
}

\section{Mansi M. Kasliwal ${ }^{1,2}$ (on behalf of the Palomar Transient Factory Collaboration)}

\author{
${ }^{1}$ Observatories of the Carnegie Institution for Science, Pasadena, CA 91101, USA \\ ${ }^{2}$ Dept. of Astrophysical Sciences, Princeton University, Princeton, NJ 08544, USA \\ email: mansi@astro.caltech.edu
}

\begin{abstract}
Until recently, the venerable field of cosmic explosions has been plagued with a glaring six-magnitude luminosity gap between the brightest novæ and the faintest supernovæ. A key science driver of the Palomar Transient Factory was a systematic search for optical transients that are fainter, faster and rarer than supernovæ. Theorists predict a variety of mechanisms to produce transients in that "gap", and observers have the best chance of finding them in the local universe. The talk presented the discoveries and the unique physics of cosmic explosions which bridge that gap between novæ and supernovæ. As Fig. 1 illustrates, there is now evidence of multiple, distinct populations of rare transients in the "gap".
\end{abstract}

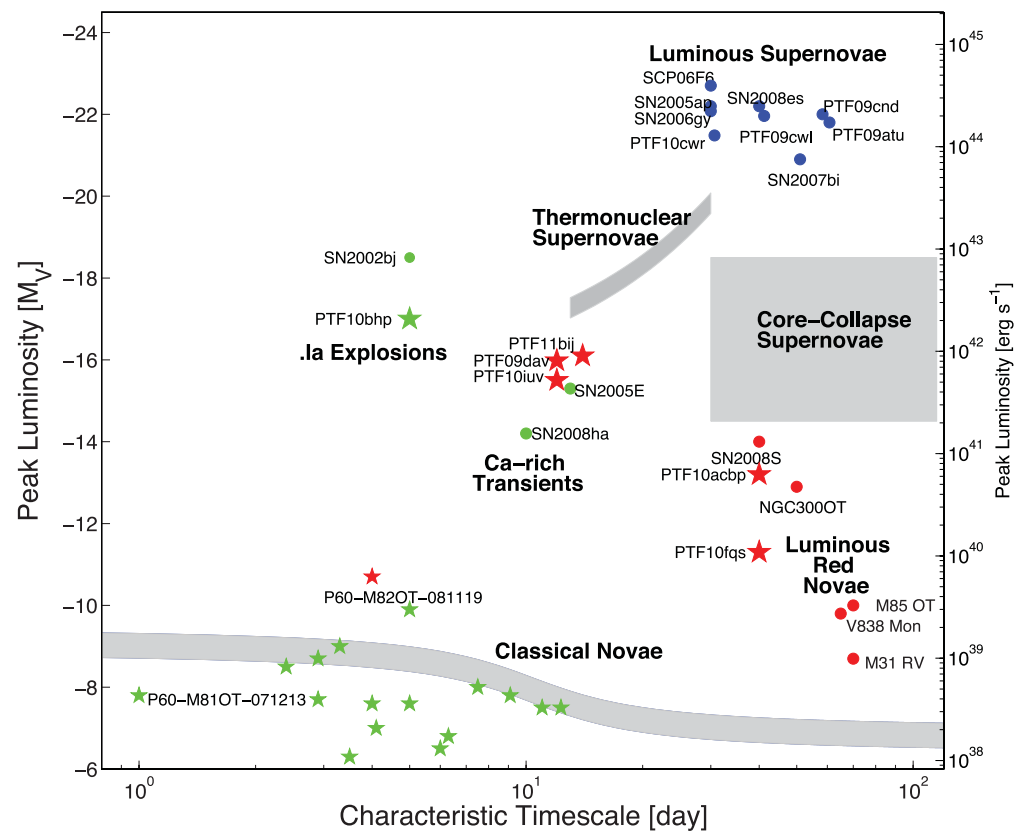

Figure 1. Framework of Cosmic Explosions expressed as peak luminosity versus characteristic time-scale. In 2005 we only knew about classical novæ and supernovæ (grey bands). In the past 5 years systematic searches, serendipitous discoveries and archival searches have uncovered a plethora of novel, rare transients. Several new classes are emerging, and the governing physics is being widely debated: luminous red novae (the electron-capture induced collapse of rapidly rotating $\mathrm{O}-\mathrm{Ne}-\mathrm{Mg}$ white dwarfs?), luminous supernovæ (magnetars or pair instability explosions?), Type Ia explosions (helium detonations in ultra-compact white-dwarf binaries), calcium-rich halo transients (helium deflagrations or fallback onto a black hole?) (from Kasliwal, M.M., 2011, PhD Thesis, California Institute of Technology). 